\title{
gु \\ Thickness dependence and the role of spin transfer torque in nonlinear giant magnetoresistance of permalloy dual spin valves
}

\author{
N. Banerjee, ${ }^{1}$ A. Aziz, ${ }^{1}$ M. Ali, ${ }^{2}$ J. W. A. Robinson, ${ }^{1}$ B. J. Hickey, ${ }^{2}$ and M. G. Blamire ${ }^{1}$ \\ ${ }^{1}$ Department of Materials Science and Metallurgy, University of Cambridge, Pembroke Street, Cambridge CB2 3QZ, United Kingdom \\ ${ }^{2}$ School of Physics and Astronomy, University of Leeds, Leeds LS2 9JT, United Kingdom
}

(Received 10 September 2010; published 2 December 2010)

\begin{abstract}
The recent discovery of nonlinear current-dependent magnetoresistance in dual spin valve devices [A. Aziz, O. P. Wessely, M. Ali, D. M. Edwards, C. H. Marrows, B. J. Hickey, and M. G. Blamire, Phys. Rev. Lett. 103, 237203 (2009)] opens up the possibility for distinct physics which extends the standard model of giant magnetoresistance. When the outer ferromagnetic layers of a dual spin valve are antiparallel, the resulting accumulation of spin in the middle ferromagnetic layer strongly modifies its bulk and interfacial spin asymmetry and resistance. Here, we report experimental evidence of the role of bulk spin accumulation in this nonlinear effect and show that interfacial spin accumulation alone cannot account for the observed dependence of the effect on the thickness of the middle ferromagnetic layer. It is also shown that spin torque acting on the middle ferromagnetic layer combined with the nonlinear effect might be useful in understanding the dynamical features associated with the nonlinear behavior.
\end{abstract}

DOI: 10.1103/PhysRevB.82.224402

PACS number(s): 85.75.-d, 85.70.Kh

\section{INTRODUCTION}

Giant magnetoresistance (GMR) in heterostructure devices consisting of ferromagnet/nonmagnet/ferromagnet (FM/NM/FM) layers results from the differential scattering of electrons, whose spins are aligned parallel (majority) or antiparallel (minority) to the local magnetic moment, ${ }^{1,2}$ by the FM layers. Consequently, the resistance of these devices depends on the relative orientation of the FM layer moments. In current-perpendicular-to-the-plane (CPP) FM/NM/FM geometries, GMR is well described by Valet-Fert ${ }^{3}(\mathrm{~V}-\mathrm{F})$ theory which is based on the Boltzmann transport equations; indeed, this theory has been applied extensively to analyze spindependent electron transport in FM/NM multilayers, FM/ $\mathrm{NM} / \mathrm{FM}$ spin valves (SVs), as well as dual SV (DSV) devices with $\mathrm{FM} 1 / \mathrm{NM} / \mathrm{FM} / \mathrm{NM} / \mathrm{FM} 3$ layers. One key assumption in the $\mathrm{V}-\mathrm{F}$ model is the transport of electrons through a constant density of states (DoS) in each layer resulting from a simplified single conduction band. Other important assumptions include: current-independent values of bulk and FM/NM interfacial resistance and scattering asymmetries. When a current passes through a FM/NM bilayer or a FM/NM/FM SV in CPP mode, the electrons are spin polarized by the FM layers due to their spin-split DoS. When the spin-polarized current from the FM layers arrive at the FM/NM interface and enters the NM, the excess electrons of one spin direction will equilibrate their spins due to the equal DoS for both spins in the NM. In dynamic equilibrium, the spin relaxation rate and hence the spin accumulation ${ }^{4}$ is equal to the spin injection rate. V-F theory assumes the spin accumulation to be small compared with the variations in DoS, which results in the DoS available for electron scattering in each layer to be constant and independent of spin accumulation.

In addition to GMR, a spin-polarized current can give rise to dynamical effects via spin transfer torque ${ }^{5}$ (STT). When a spin-polarized current encounters a magnetic thin film with a noncollinear magnetization, a part of the spin angular mo- mentum is absorbed. As the spins of the electrons rotate to align with the magnetization of the second layer, they exert an equal and opposite torque on the ferromagnet (i.e., STT). For sufficiently large current densities of the order $10^{7} \mathrm{~A} / \mathrm{cm}^{2}$, the STT can be enough to reorient the FM layer magnetizations in the opposite direction. In an asymmetric FM1/NM/FM2 spin valve in which the thickness of FM1 $>$ FM2, the spin-polarized current generated by FM1 exerts STT on the moment of FM2. If FM2 is now sandwiched between two identically thick FM layers with antiparallel alignment to form a DSV, the spin accumulation in the middle (FM2) layer is predicted to be three times larger than that produced by an asymmetric spin valve as shown by Berger. $^{6}$ The high-spin accumulation in addition to the fact that both interfaces are available for the middle layer to generate torque, potentially reduces the critical current density required for STT switching.

Recently, Aziz et al. ${ }^{7}$ discovered a different aspect of this high-spin accumulation in the middle FM layer of a DSV. It was shown that for antiparallel alignment of the outer two FM layers (FM1 and FM3), the resistance of the DSV structure depended on the current and on the direction of magnetization of the middle FM2 layer. This implies that the states $\uparrow \uparrow \downarrow$ and $\uparrow \downarrow \downarrow$ have different (current-dependent) resistances, where the arrows indicate the magnetic configuration of FM1, FM2, and FM3 layers. Conventional V-F theory predicts identical resistance for $\uparrow \uparrow \downarrow$ and $\uparrow \downarrow \downarrow$ states as well as the magnetoresistance to be independent of current, which indicates that this observed behavior cannot be explained within the current understanding of GMR.

A possible explanation of the nonlinear current-dependent behavior can be found by taking into account the band structure of the FM2 layer. ${ }^{7}$ The assumption of constant transport parameters in V-F theory breaks down under the intense spin accumulation seen in DSVs for antiparallel outer FM layer orientation. If there exists a large gradient in the DoS near the Fermi level (as opposed to the constant DoS assumption in $\mathrm{V}-\mathrm{F}$ model), the high level of spin accumulation can 
modify the DoS that the electron "sees" during transport. This in effect changes the scattering rates and modifies the transport parameters stated above. As the high-spin accumulation changes with the magnitude of the current flowing through the DSV, the magnetoresistance is expected to be modified with current as observed in Ref. 7.

A recent analysis by Baláž and Barnaś ${ }^{8}$ shows that the modification of spin asymmetries and resistivities (as a function of current and hence spin accumulation) at the interface and bulk leads to similar nonlinear GMR effects as observed experimentally. To analyze the effect in more detail, the relative contribution from the bulk and interface should be evaluated. Hence, in this paper we explain the relative contribution from the FM/NM interfaces and the bulk FM layers by varying the bulk spin accumulation. We have achieved this by varying the thickness of the middle FM layer (FM2) which in effect keeps the interfacial spin accumulation constant but changes the bulk spin accumulation. In addition, we also report the observation of additional dynamical features associated with the nonlinear effect and show that spin torque acting on FM2 might be useful in understanding them.

\section{EXPERIMENTAL}

The DSV structures were sputter deposited on unheated $10 \times 5 \mathrm{~mm}^{2} \mathrm{Si} / \mathrm{SiO}_{2}$ substrates having an active layer sequence of $\mathrm{Co}_{90} \mathrm{Fe}_{10}(6 \mathrm{~nm}) / \mathrm{Cu}(4 \mathrm{~nm}) / \mathrm{Py}(x \mathrm{~nm}) / \mathrm{Cu}$ $(4 \mathrm{~nm}) / \mathrm{Co}_{90} \mathrm{Fe}_{10}(6 \mathrm{~nm}) / \operatorname{IrMn}(10 \mathrm{~nm})$ in a chamber with a base pressure of less than $5 \times 10^{-8}$ mbar. Several series of samples were prepared with each sample having a different Py $\left(\mathrm{Ni}_{80} \mathrm{Fe}_{20}\right)$ layer thickness varying from 1 to $5 \mathrm{~nm}$. Each respective sample will be referred to as DSV $(x)$. Although the top CoFe layer is exchange biased via the IrMn pinning layer, the MR curves did not show significant bias. Above and below the DSV structure we deposited 200-nm-thick $\mathrm{Cu}$ electrodes. We also deposited a $5 \mathrm{~nm}$ layer of Ta at the bottom and at the top to prevent oxidation of the $\mathrm{Cu}$ contacts and to minimize ion-beam damage from subsequent processing steps. Using standard optical lithography and Ar-ion milling, a series of $4-\mu \mathrm{m}$-wide tracks were created which were connected to large area contact pads for four terminal electrical measurements. By using a focused ion beam nanomachining technique, a number of nanopillar devices were fabricated in the 4- $\mu$ m-wide tracks (as described elsewhere). ${ }^{9,10}$ This was achieved in a four-step process: (1) narrowing of the 4- $\mu \mathrm{m}$-wide track to a width of $\sim 500 \mathrm{~nm}$ using a beam current of $150 \mathrm{pA}$ [Fig. 1(a)]; (2) using a reduced beam current of $11 \mathrm{pA}$, the track width was further reduced to $\sim 300 \mathrm{~nm}$; (3) sample is rotated to an angle of $85^{\circ}$ with respect to the ion beam and two side cuts are made, forming a CPP nanopillar device as illustrated in Fig. 1(b); (4) sample is rotated back to $0^{\circ}$ [as shown in Fig. 1(a)] and the track width is again narrowed with an $11 \mathrm{pA}$ beam to $\sim 200 \mathrm{~nm}$. This final processing step gently etches the sidewalls and removes material implanted with Ga. Up to a maximum of 14 devices were fabricated on a single chip. Devices were measured at room temperature using a lock-in technique with a frequency of $3.46 \mathrm{kHz}$ and an alternating

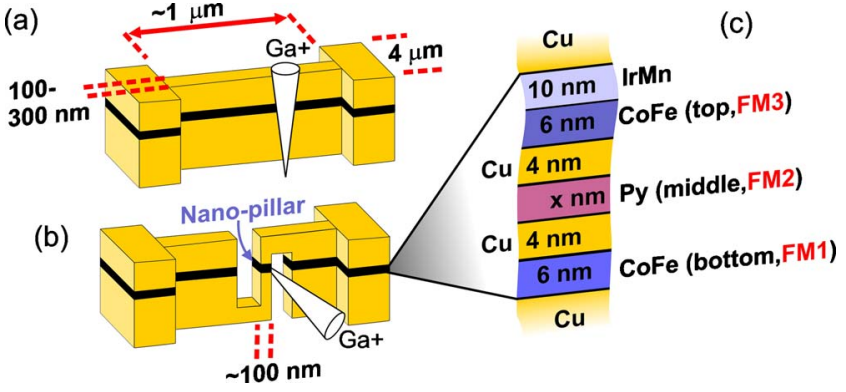

FIG. 1. (Color online) Nanopillar device fabrication using a focused beam of $\mathrm{Ga}$ ions: (a) $4-\mu \mathrm{m}$-wide tracks are narrowed by milling with the beam at $90^{\circ}$ to the surface plane; (b) the sample is rotated to at an angle of $85^{\circ}$ with respect to the surface plane and two side cuts are made (defining the nanopillar geometry and current path); and (c) detail of the DSV active layer sequence that is sandwiched between top and bottom 200-nm-thick Cu electrodes.

current amplitude of $I_{\mathrm{ac}}=100 \mu \mathrm{A}$ superimposed on a bias current $\left(I_{\mathrm{dc}}\right)$. A positive value of $I_{\mathrm{dc}}$ corresponds to electrons flowing from the $\mathrm{IrMn} / \mathrm{CoFe}$ (FM3) layer to the lower $\mathrm{CoFe}$ (FM1) layer.

\section{RESULTS}

The DSV devices consist of three FM layers and so a number of different magnetic states can be obtained by changing the relative orientation of the magnetic moments of the individual layers. To analyze these states, we first examine the full resistance vs magnetic field loop (GMR loop) of a DSV and the different magnetic states associated with them.

Figure 2 shows the GMR loop for DSV (3) at 0 dc bias current. At $\pm 100 \mathrm{mT}$ all three FM layers point in the same direction. As the applied field is lowered, at $\approx 25 \mathrm{mT}$ the

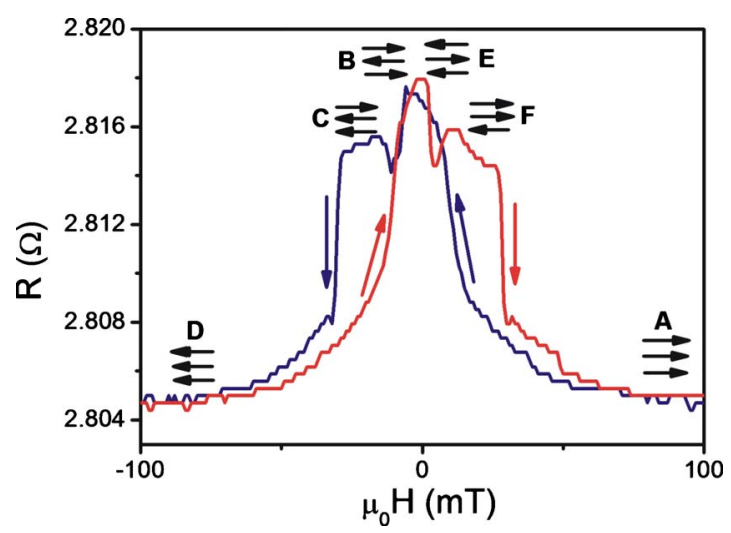

FIG. 2. (Color online) Low current full resistance vs magnetic field loop at room temperature of a $230 \times 280 \mathrm{~nm}^{2} \mathrm{DSV}$ which has the following active layer sequence: $\mathrm{Co}_{90} \mathrm{Fe}_{10}(6 \mathrm{~nm}) /$ $\mathrm{Cu}(4 \mathrm{~nm}) / \mathrm{Py}(3 \mathrm{~nm}) / \mathrm{Cu}(4 \mathrm{~nm}) / \mathrm{Co}_{90} \mathrm{Fe}_{10}(6 \mathrm{~nm}) / \operatorname{IrMn}(10 \mathrm{~nm})$ [DSV (3)]. The blue (dark gray) and red (light gray) data correspond, respectively, to decreasing and increasing field sweeps as indicated by the arrows. The bold (black) arrows labeled A-F indicate the direction of the magnetic layer moments of FM1, FM2, and FM3 layers. 

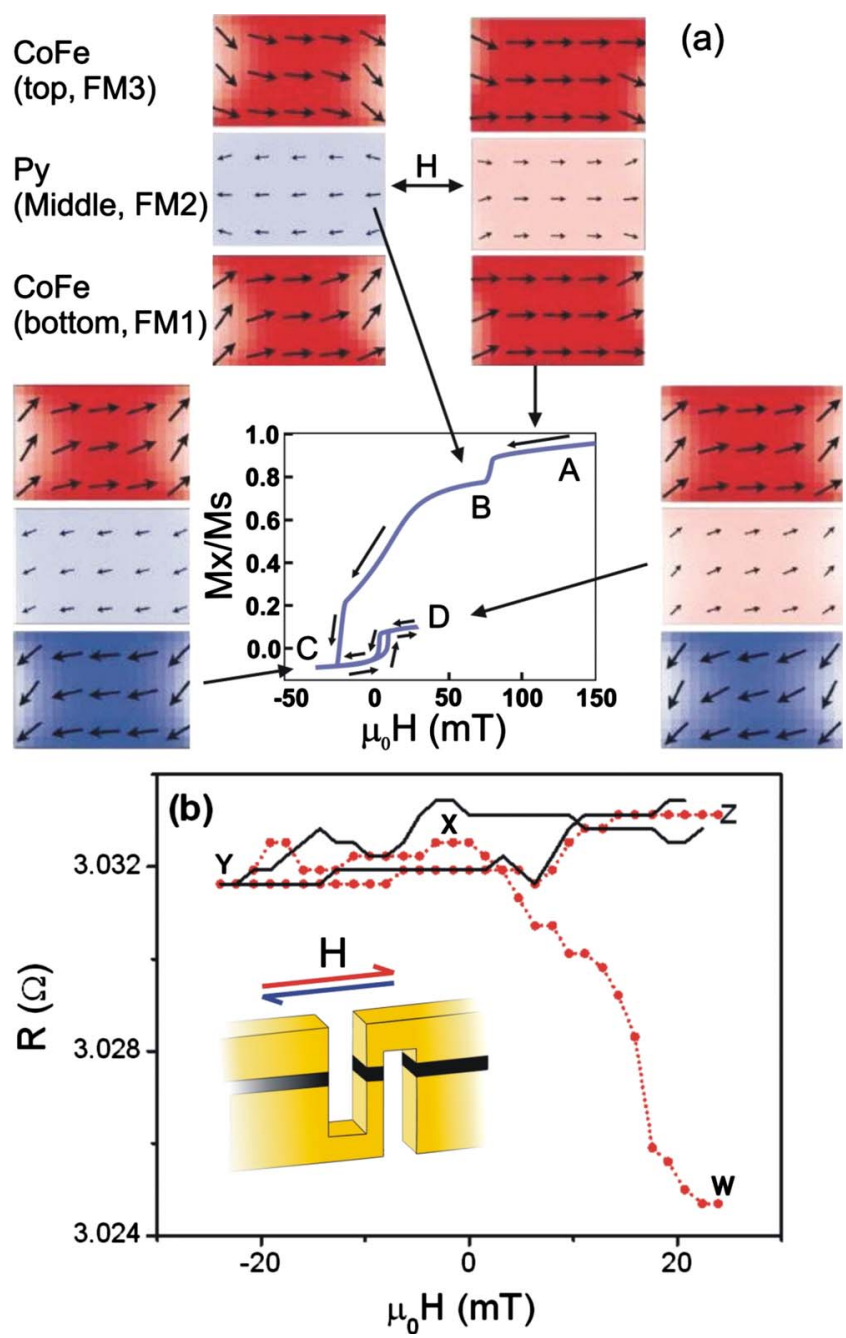

FIG. 3. (Color online) (a) Micromagnetic simulations at zero dc bias of DSV (2) in a cycled magnetic field to achieve the necessary ferromagnetic layer configurations for high-spin accumulation (adapted from Ref. 7). The magnetic states of the individual layers are shown as plan views, corresponding to different points of the magnetic cycle (taken from Ref. 7). (b) Shows the actual switching process in a device subjected to a similar magnetic cycle as in (a) for zero dc bias. In both (a) and (b) the magnetic field is applied parallel to the long axis of a device, as illustrated in the inset of (b).

middle Py layer rotates due to its magnetostatic interaction with FM1 and FM3 layers. Point B in the figure represents this state, which has the highest resistance since it corresponds to two antiparallel spin valves in series. The small drop in resistance around point $\mathrm{C}$ indicates the reversal of the lower $\mathrm{CoFe}$ layer which is then followed by the rotation of the top CoFe layer giving the lowest resistance state of the device (point D).

Micromagnetic simulations ${ }^{11}$ of these DSV structures show that other magnetic states can be created in addition to the standard ones described above. In Fig. 3(a) the plan views of magnetization states of FM1, FM2, and FM3 layers of DSV (2) at different magnetic fields applied along the long axis are shown. Although, as an example a thickness of $2 \mathrm{~nm}$ is used for the middle Py layer in the simulation, similar states are obtained with other Py layer thicknesses. A bias

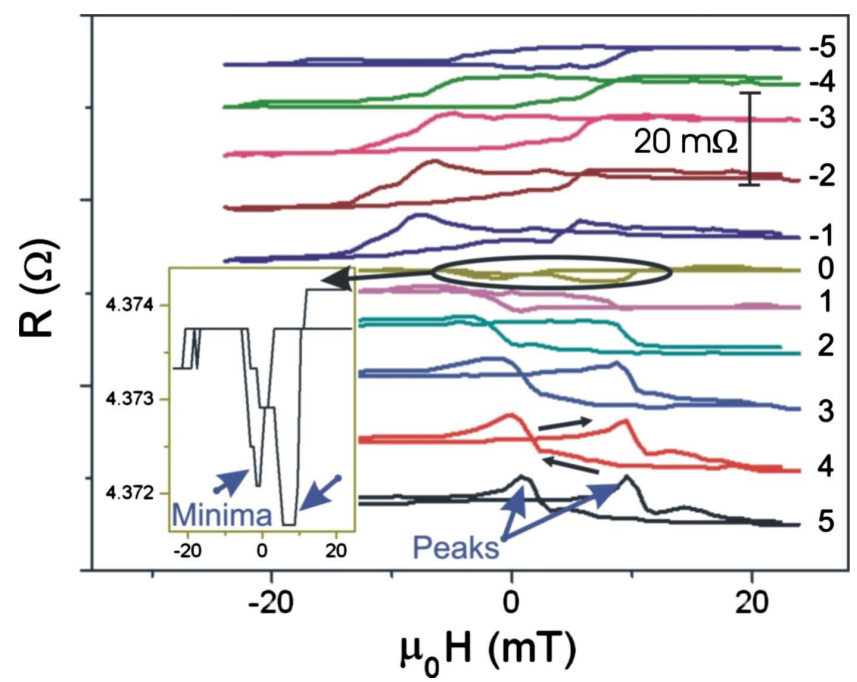

FIG. 4. (Color online) Minor loops as a function of applied bias current for DSV (2). Numbers correspond to the applied dc bias current in milliampere units. The device is cycled between +22 to $-22 \mathrm{mT}$ and the magnetic field sweep directions are indicated by arrows. Positive current denotes electron flow from $\mathrm{IrMn} / \mathrm{CoFe}$ to $\mathrm{CoFe}$. The inset shows the minor loop at zero dc bias with the resistance minima indicated. All the curves are vertically offset for clarity.

field of $10 \mathrm{mT}$ is applied to the top CoFe layer. For our simulation, the pillar structure is divided into cells of size $10 \times 10 \times 2 \mathrm{~nm}^{3}$. Saturation magnetization, exchange coefficient, and uniaxial anisotropy of $\mathrm{CoFe}$ are set to 1.8 $\times 10^{6} \mathrm{~A} \mathrm{~m}^{-1}, 3 \times 10^{-11} \mathrm{~J} \mathrm{~m}^{-1}$, and $-1.5 \times 10^{4} \mathrm{~J} \mathrm{~m}^{-3}$, respectively. The corresponding values for $\mathrm{NiFe}$ are 0.86 $\times 10^{6} \mathrm{~A} \mathrm{~m}^{-1}, 1.3 \times 10^{-11} \mathrm{~J} \mathrm{~m}^{-1}$, and $-3 \times 10^{3} \mathrm{~J} \mathrm{~m}^{-3}$, respectively. The damping coefficient is set to 0.5 , which allows rapid convergence. The states represented in the simulation refer to zero dc bias condition. Starting from a high field of $150 \mathrm{mT}$ we see that all the three FM layers point in the direction of the external applied field. When the external field is reduced to around $70 \mathrm{mT}$ [point $\mathrm{B}(\uparrow \downarrow \uparrow)]$ the middle Py layer reverses its orientation under the action of the magnetostatic fields arising from the two CoFe layers. On crossing the zero field the applied field in the negative direction now switches the bottom CoFe layer [point $C(\uparrow \downarrow \downarrow)$ ], producing a magnetic state where the outer FM layers are pointing in the opposite direction. If the DSV is cycled between the points $\mathrm{C}$ and $\mathrm{D}(\uparrow \uparrow \downarrow)$ the Py layer switches direction without affecting the magnetic configuration of the CoFe layers. The experimental resistive changes at zero dc bias are shown in Fig. 3(b) for a DSV (2). The dashed line starts at around $+17 \mathrm{mT}$ [point $\mathrm{W}(\uparrow \uparrow \uparrow)]$ which represents a magnetic state where the three FM layers are still parallel with the middle layer just starting to rotate and the resistance of the device is low. But when the external magnetic field is decreased, due to the magnetostatic interaction from the outer $\mathrm{CoFe}$ layers the middle Py layer rotates. This produces a high resistance state represented by the point $X(\uparrow \downarrow \uparrow)$ in Fig. 3(b). Further reduction in the external magnetic field in the negative direction now rotates the bottom CoFe layer and the device resistance drops from point $\mathrm{X}(\uparrow \downarrow \uparrow)$ to point $\mathrm{Y}$ 


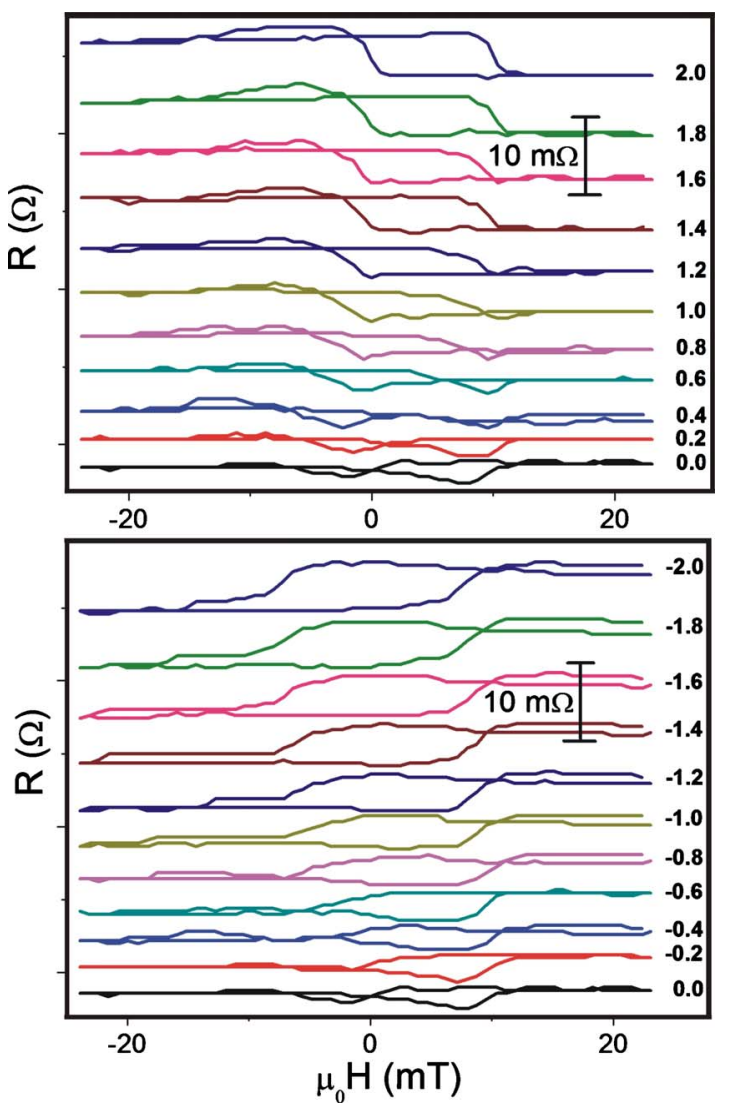

FIG. 5. (Color online) Minor loops as a function of applied dc bias current for DSV (2) with a current step size of $0.2 \mathrm{~mA}$. The maximum range of the applied current is now decreased to +2 to $-2 \mathrm{~mA}$ in order to study the current dependence of the resistance minima (numbers in milliampere units). All the curves are vertically offset for clarity.

$(\uparrow \downarrow \downarrow)$. The resistance change on cycling the device between the points marked by $\mathrm{Y}$ and $\mathrm{Z}$ [which represents the minor loop C-D in Fig. 3(a)] is represented by the solid line. Magnetically this represents the rotation of the middle Py layer only. Although, for most devices the resistance states marked by $\mathrm{Y}$ and $\mathrm{Z}$ are almost identical at zero dc bias (solid black line), sometimes there is a small shift as seen here. In such cases, states represented by points $\mathrm{Y}$ and $\mathrm{Z}$ have identical resistances at small dc biases on the order of $\pm 200 \mu \mathrm{A}$ rather than at zero bias. From the solid line loop it is clear the resistance change is very small during this cycle (on the order of $0.8 \mathrm{~m} \Omega$ ). By symmetry of these two states [marked by points $\mathrm{C}$ and $\mathrm{D}$ in Fig. 3(a)], it can be concluded from V-F theory that the resistance of the device should be identical. In other words, the orientation of the Py layer should not make a difference to the total resistance of the nanopillar. In fact this is what we observe Fig. 3(b) where the change in resistance on reversal of only the Py layer is negligible. The key result presented in Aziz et al. ${ }^{7}$ was that biasing the structure with dc current makes the resistance of these two states distinct. The resistance of the structure now depends on the orientation of the Py layer and the sign of the resistance change depends on the direction of this dc bias current.

Figure 4 shows a series of such minor loops at various bias current values for DSV (2). It is clear from the figure

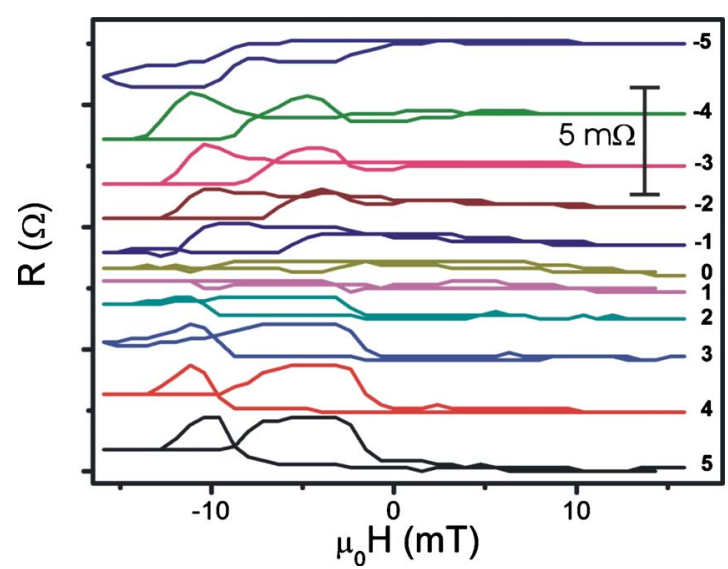

FIG. 6. (Color online) A series of minor loops as a function of applied dc bias for DSV (3), showing the appearance of peaks near the reversal field of Py (FM2). The peaks appear in addition to the resistance minima at higher currents (numbers in milliampere units). The offset in switching fields observed here is irreproducible and is not a particular feature of these devices. All the curves are vertically offset for clarity.

that on application of a bias current the resistance change between the two states is quite dramatic and asymmetric with respect to the direction of current flow. It was suggested in Ref. 7 that this dramatic modification of minor loop arises from high-spin accumulation in the middle Py layer.

Closer inspection of Fig. 4 reveals some structures which possibly have a dynamic origin. Sharp resistance minima are observed, which are also shown separately as inset of Fig. 4 for the zero-bias minor loop, close to the region where reversal occurs at low currents of up to $2 \mathrm{~mA}$. In order to investigate these resistance minima, minor loops were measured with smaller current steps in the range of -2 to $+2 \mathrm{~mA}$ (Fig. 5). We see the resistance drops disappear with increasing current. But beyond the low current range as one moves to higher currents, additional sharp peaks appear close to the reversal region, even after the resistance minima have disappeared. One possible reason for these peaks could be STT at higher current; to test the reproducibility of these features similar minor loop experiments were done with DSV (3) having a $3 \mathrm{~nm}$ Py as the middle layer. The minor loops in Fig. 6, show similar resistance minima at low currents and appearance of peaks at higher currents.

To investigate the role of STT as a possible origin of the peaks in minor loops, a measurement of the differential resistance $(R)$ as a function of current $\left(I_{\mathrm{dc}}\right)$ was performed for DSV (3) as depicted in Fig. 7(a). Initially the device was saturated by applying a field of $200 \mathrm{mT}$. Next the device is subjected to the following field cycle: $200 \rightarrow 12 \rightarrow-12 \rightarrow 12$ $\rightarrow-10 \mathrm{mT}$. The main aim of a complete cycle around 12 $\mathrm{mT}$ is to align the outer two layers antiparallel to each other and ending the cycle at $-10 \mathrm{mT}$ results in the Py layer placed close to the point of reversal; keeping the outer two CoFe layers fixed, the device is now held at this field and the resistance monitored as a function of applied dc bias current. Between -3 and $-5 \mathrm{~mA}$ we see a loop in the $R$ vs $I_{\mathrm{dc}}$ plot showing a clear switching region near $-5 \mathrm{~mA}$. With decreasing current magnitude the resistance difference between the 


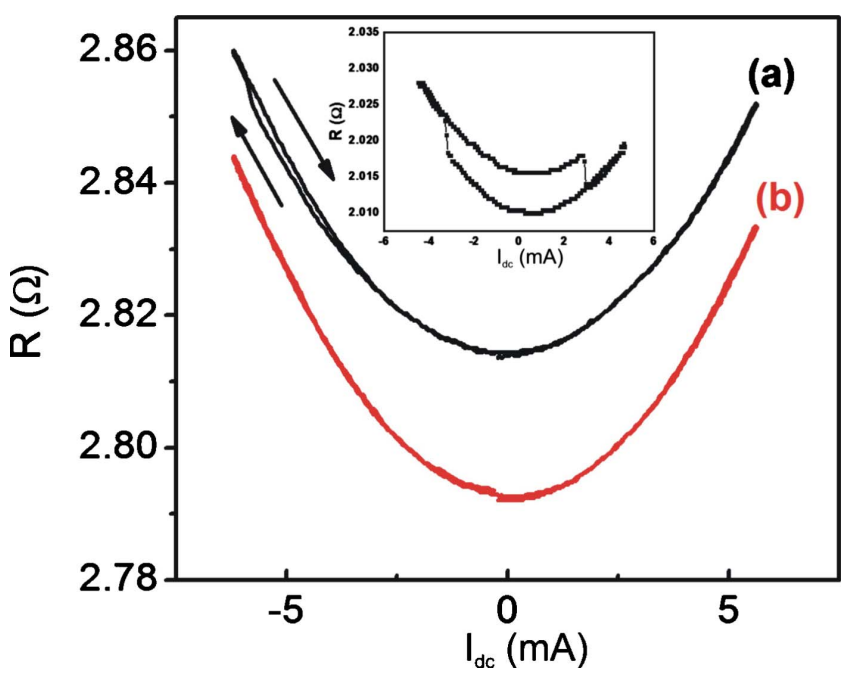

FIG. 7. (Color online) (a) Differential resistance vs $I_{\mathrm{dc}}$ for DSV (3) with device dimensions $230 \times 280 \mathrm{~nm}^{2}$ measured at $-12 \mathrm{mT}$ with CoFe layers antiparallel. Spin torque switching is seen at negative currents, indicating that the peaks appearing in minor loops for these devices may originate from spin torque effects. The arrows in (a) indicate the direction of the applied current sweep. (b) Differential resistance as a function of $I_{\mathrm{dc}}$ for the same device measured at +16 mT with the outer CoFe layers parallel. Absence of any spin torque switching suggests that this effect only arises when the outer CoFe layers are antiparallel. An offset of $-0.2 \Omega$ has been applied to the second curve for clarity. Inset: comparison data showing a typical spin torque switching in an asymmetric spin valve device having an active layer structure of $\mathrm{CoFe}(96 \mathrm{~nm}) / \mathrm{Cu}(6 \mathrm{~nm}) / \mathrm{CoFe}$ (3 $\mathrm{nm})$.

original state and the switched state gradually diminishes and becomes zero close to $-3.5 \mathrm{~mA}$. If, instead of executing a minor loop cycle between +12 and $-12 \mathrm{mT}$ the magnetic field is reduced from 200 to $+15 \mathrm{mT}$, the outer layers retain their original orientation. This represents a configuration $(\uparrow \downarrow \uparrow)$ where the outer CoFe layers are parallel to each other but the middle Py layer rotates due to the magnetostatic interaction with the CoFe layers. A $R$ vs $I_{\mathrm{dc}}$ measurement [Fig. 7(b)] shows no spin torque switching signal for such a magnetic configuration, as the torque exerted on the middle Py layer by spin-polarized electrons passing through the bottom $\mathrm{CoFe}$ layer and the reflected electrons from the top $\mathrm{CoFe}$ layer are in opposition. Only for an antiparallel outer $\mathrm{CoFe}$ layer configuration are the torque exerted on the middle Py layer by the transmitted and reflected spin-polarized electrons in conjunction. In order to determine the underlying cause of the resistance change between $\uparrow \uparrow \downarrow$ and $\uparrow \downarrow \downarrow$ states, it is important to ascertain the role of the interplay between interfacial and bulk spin accumulation. This is achieved by noting the resistance change in the minor loops with bias current for different Py layer thicknesses. Figure 8 shows the change in resistance-area product $(A \Delta R)$ with current as a function of Py layer (FM2) thickness. There is not much difference between $1 \mathrm{~nm}$ and $2 \mathrm{~nm}$ samples. The $3 \mathrm{~nm}$ curve lies below the ones for $1,1.5$, and $2 \mathrm{~nm}$ and this is true for all the devices examined. A series of minor loops are shown in Fig. 9 for DSV (5). It is clearly seen there is no detectable minor loop modification on dc biasing the structure.

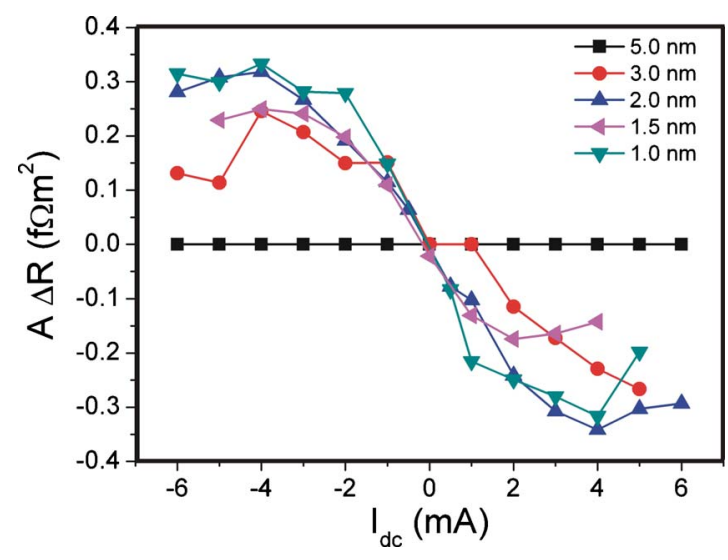

FIG. 8. (Color online) The change in the resistance-area product with current and middle Py layer (FM2) thickness. For DSV devices with a Py thickness of $5 \mathrm{~nm}$ the variation $A \Delta R$ is negligible with no apparent correlation with the current direction. Each datum point represents the average value of $A \Delta R$ from many devices and chips measured.

\section{DISCUSSION}

GMR in a SV arises because of the differential scattering of majority- and minority-spin electrons in the bulk of a FM and at the FM/NM interface which are characterized by bulk and interfacial scattering asymmetries $(\beta$ and $\gamma)$. For the MR to be dependent on current (as in Fig. 4) we require either or both of $\beta$ and $\gamma$ to be current dependent.

The recent analysis in Ref. 8 has taken into account this modification in $\beta$ and $\gamma$ as well as the bulk and interfacial resistivities by introducing phenomenological constants that describe band-structure effects and parameters which take spin accumulation into consideration. The resistance of the structure, calculated for arbitrary noncollinear magnetic configurations ${ }^{12,13}$ with the above-mentioned modifications indeed leads to changes in resistance with Py orientation that

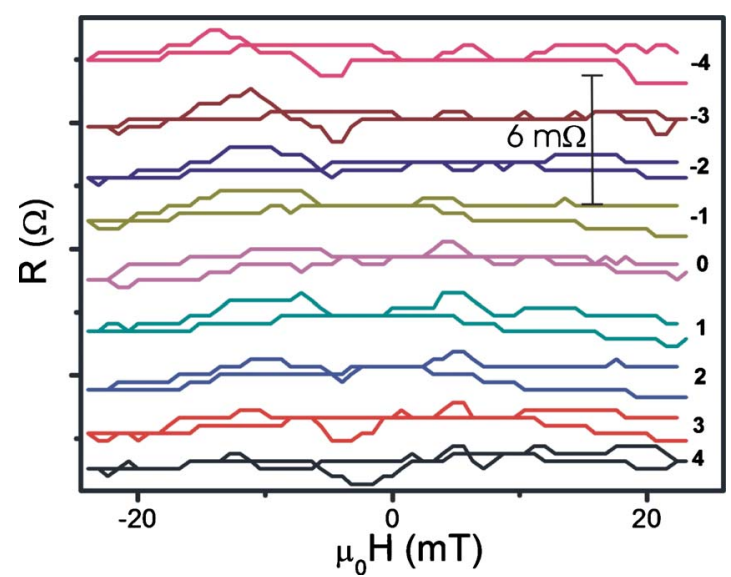

FIG. 9. (Color online) Minor loops for a device with a 5-nmthick layer of Py showing no modification with current amplitude (numbers in milliampere units). Data from some devices show very little changes which are not consistent with direction of current flow suggesting that the nonlinear effect disappears for Py thicknesses (FM2) close to $5 \mathrm{~nm}$. All the curves are vertically offset for clarity. 
have been observed experimentally. Interfacial and bulk contributions of this effect were studied separately by changing the interfacial parameters while keeping the bulk parameters fixed and vice versa. The interfacial contribution leads to a thickness dependence, with the effect decreasing on increasing Py layer thickness. The bulk contribution shows no such thickness dependence.

In addition to the modification of minor loops with current, their analysis also reveals the experimentally observed resistance minima, which were seen in Fig. 5. In order to perform the analysis, they solved the Landau-Lifshitz-Gilbert equation to study the time evolution of the spin moment of the middle layer. According to the authors, during the switching of the magnetization between two collinear orientations, it executes a precessional motion and comes out of plane. This precessional motion of the magnetization vector changes the spin accumulation as well as the resistance of the structure, giving rise to a resistance minimum at low current density near the reversal regions. The effective field used in their simulation includes the external applied field, the anisotropy field, the demagnetization field, and a stochastic Gaussian field representing the thermal fluctuations. Spin torque was not included in the simulations.

STT switching of a conventional asymmetric SV appears as two abrupt changes in a plot of resistance vs current which corresponds to transitions between parallel and antiparallel configurations of the FM layers as shown in the inset of Fig. 7(a). Although the larger spin accumulation of a DSV with antiparallel outer layers has been predicted to enhance STT, ${ }^{6}$ the configurations between which the middle layer is switched would have identical GMR values on the basis of the V-F model. However, nonlinearity in GMR would mean that the resistances of the $\uparrow \uparrow \downarrow$ and $\uparrow \downarrow \downarrow$ states, although degenerate at zero current, would diverge with increasing current. This provides a simple explanation for the form of the loop shown in Fig. 7(a): for negative currents, two distinct resistance states are observed which diverge with increasing current magnitude, until the device switches between the two states. If the reverse switching process (under positive current) occurs at low current then it will not be visible as a resistance change. This apparently anomalous STT behavior provides further evidence for the nonlinear GMR effect in a DSV.

The spin accumulation that gives rise to the nonlinear effect is not only a function of current as explained above but a function of Py layer thickness as well. The spin accumulation at the FM/NM interface penetrates into the FM and NM and decays exponentially with a characteristic length scale (the spin-diffusion length) away from the interface into the bulk. Therefore, the high-spin accumulation in the middle Py layer is expected to decay with increasing Py thickness. So the $\Delta R$ at a particular current will decrease with increasing Py thickness (Fig. 8). The modification of the DoS available for electron scattering in Py depends on the bulk spin accumulation. Since the spin accumulation varies with distance from the interface, the average spin accumulation is inversely proportional to the Py layer thickness $d$. Increasing $d$ therefore, reduces average spin accumulation in the bulk which in turn reduces the modification of the DoS at a particular bias current. Hence, for the thicknesses of the middle
Py layer that are equal to or greater than the spin diffusion length, the zero current situation is not much different from the high-bias current one. This makes the resistance of a device with a thicker middle layer insensitive to Py layer rotation under dc bias current. But in all of these devices with variable middle FM layer thickness, the interfacial spin accumulation remains unchanged. Interfacial resistance arises due to spin-sensitive scattering potential present at the interface. ${ }^{14}$ Analysis of spin-sensitive interfacial resistance for CPP transport by Barnaś and Fert ${ }^{15}$ shows that interfacial resistance arising due to potential steps between FM/NM interface and impurity or defect induced scattering potential at the interface depends on the FM band structure and the DoS at $E_{F}$. On reducing the bulk spin accumulation, the DoS available to electrons flowing through the middle FM layer is not much different from that of the zero current case. Thus, at a particular current, increasing the thickness of the middle FM layer would mean a lower degree of modification of the scattering asymmetries and resistivities. When the middle FM thickness exceeds the spin-diffusion length of the material we expect the average spin accumulation in the layer to fall drastically. This implies there is very little or no modification of the bulk DoS from that of the equilibrium value at zero current and the device resistance is same for two antiparallel states $\uparrow \uparrow \downarrow$ and $\uparrow \downarrow \downarrow$. Analysis by Tysmbal and Pettifor $^{16}$ shows that spin-independent interfacial scattering combined with spin-polarized bulk electronic band structure provides a more realistic approach for understanding GMR better. Adopting this perspective to understand the relative contribution of the bulk FM layers and the FM/NM interfaces in the nonlinear effect, shows modification of DoS of bulk FM is more important to understanding the dependence of this effect on the thickness of the middle Py layer than interfacial spin accumulation.

We observe from Figs. 8 and 9 that the nonlinear GMR effect disappears around a Py thickness of $\sim 5 \mathrm{~nm}$ which is approximately the spin-diffusion length of Py (Ref. 17) and this agrees with our original argument given above. The variation in resistance of the Py between thickness 2 and 5 nm would not be significant enough to mask the effect completely and can be ruled out as the cause for why the effect disappears. Significant changes in resistance occur due to Joule heating of the devices during dc bias measurements at high current densities. A considerable portion of the resistance change due to Joule heating with dc bias current originates from the thick top and bottom $\mathrm{Cu}$ leads, which makes it difficult to isolate the resistance change coming solely from the device due to heating. For this reason, we have measured devices with varying dimensions which in effect changes the amount of Joule heating associated with them. In spite of the change in device dimensions for a particular Py layer thickness, the nonlinear effect is unaffected and the $A \Delta R$ product remains same for these devices. This observation supports the fact that the resistance increase cannot be an underlying cause for the observed thickness dependence of the effect and its subsequent disappearance around a Py layer thickness of $\sim 5 \mathrm{~nm}$. We therefore believe, bulk spin accumulation and hence the spin-diffusion length has a significant role to play in this effect. At thicknesses well below the spin-diffusion length of Py (when the average spin accumulation is high), 
both the interface and bulk has a role to play, but for greater thicknesses the bulk spin accumulation becomes the governing factor that controls both the effect from the interfaces as well as from the bulk.

\section{CONCLUSION}

We have shown that the nonlinear GMR effect in DSV devices is primarily controlled by a modification to the bulk DoS. High-spin accumulation achieved in DSV structures results in a significant change in scattering asymmetries and resistivities due to electron transport through modified DoS. Although interfacial spin accumulation is the larger effect, it cannot account for all the results, especially the FM layer thickness dependence of the nonlinear GMR effect. Finally, we have also shown that spin transfer torque combined with the nonlinear effect arising from high-spin accumulation might be necessary to explain the appearance of peaks in the minor loops at high current densities.

\section{ACKNOWLEDGMENTS}

J.W.A.R. acknowledges support by St John's College, Cambridge. N.B. wishes to thank St. John's College, Cambridge for financial support. This work was funded by the UK EPSRC through the Spin@RT consortium.
${ }^{1}$ M. N. Baibich, J. M. Broto, A. Fert, F. Nguyen Van Dau, F. Petroff, P. Etienne, G. Creuzet, A. Friederich, and J. Chazelas, Phys. Rev. Lett. 61, 2472 (1988).

${ }^{2}$ G. Binasch, P. Grünberg, F. Saurenbach, and W. Zinn, Phys. Rev. B 39, 4828 (1989).

${ }^{3}$ T. Valet and A. Fert, Phys. Rev. B 48, 7099 (1993).

${ }^{4}$ M. Johnson and R. H. Silsbee, Phys. Rev. B 35, 4959 (1987).

${ }^{5}$ J. Slonczewski, J. Magn. Magn. Mater. 159, L1 (1996).

${ }^{6}$ L. Berger, J. Appl. Phys. 93, 7693 (2003).

${ }^{7}$ A. Aziz, O. P. Wessely, M. Ali, D. M. Edwards, C. H. Marrows, B. J. Hickey, and M. G. Blamire, Phys. Rev. Lett. 103, 237203 (2009).

${ }^{8}$ P. Baláž and J. Barnaś, Phys. Rev. B 82, 104430 (2010).

${ }^{9}$ C. Bell, G. Burnell, D.-J. Kang, R. H. Hadfield, M. J. Kappers, and M. G. Blamire, Nanotechnology 14, 630 (2003).
${ }^{10}$ M. C. Wu, A Aziz, J. D. S Witt, M. C. Hickey, M. Ali, C. H. Marrows, B. J. Hickey and M. G. Blamire, Nanotechnology 19, 485305 (2008).

${ }^{11}$ See http://math.nist.gov/oommf, Version 1.2.0.4 was used.

${ }^{12}$ M. Gmitra and J. Barnaś, Phys. Rev. B 79, 012403 (2009).

${ }^{13}$ J. Barnaś, A. Fert, M. Gmitra, I. Weymann, and V. K. Dugaev, Phys. Rev. B 72, 024426 (2005).

${ }^{14}$ J. Barnaś, A. Fuss, R. E. Camley, P. Grünberg, and W. Zinn, Phys. Rev. B 42, 8110 (1990).

${ }^{15}$ J. Barnaś and A. Fert, J. Magn. Magn. Mater. 136, 260 (1994).

${ }^{16}$ E. Yu. Tsymbal and D. G. Pettifor, Phys. Rev. B 54, 15314 (1996).

${ }^{17}$ S. Dubois, L. Piraux, J. M. George, K. Ounadjela, J. L. Duvail, and A. Fert, Phys. Rev. B 60, 477 (1999). 